\title{
Corrigendum
}

\section{A randomized controlled comparison of integrative cognitive-affective therapy (ICAT) and enhanced cognitive-behavioral therapy (CBT-E) for bulimia nervosa - CORRIGENDUM}

\section{S. A. Wonderlich, C. B. Peterson, R. D. Crosby, T. L. Smith, M. H. Klein, J. E. Mitchell and S. J. Crow}

doi:10.1017/S0033291713001098, first published online by Cambridge University Press 23 May 2013.

In the above publication by Wonderlich et al. a measure in the study has been scored incorrectly. This has resulted in a small difference in the values given for the rows corresponding to DERS Totals in tables 3 and 4.

Below are the corrected versions of table 3 and table 4 .

Table 3. Treatment Outcome by Treatment Group

\begin{tabular}{|c|c|c|c|c|c|c|}
\hline \multirow[b]{2}{*}{ Primary Outcomes } & \multicolumn{2}{|l|}{ Pretreatment } & \multicolumn{2}{|c|}{ End of Treatment } & \multicolumn{2}{|c|}{ 4-Month Follow-up } \\
\hline & $\begin{array}{l}\text { ICAT } \\
(\mathrm{n}=40)\end{array}$ & $\begin{array}{l}\text { CBT-E } \\
(\mathrm{n}=40)\end{array}$ & $\begin{array}{l}\text { ICAT } \\
(n=40)\end{array}$ & $\begin{array}{l}\text { CBT-E } \\
(n=40)\end{array}$ & $\begin{array}{l}\text { ICAT } \\
(n=40)\end{array}$ & $\begin{array}{l}\text { CBT-E } \\
(\mathrm{n}=40)\end{array}$ \\
\hline $\begin{array}{l}\text { OBE episodes (mean, SD) } \\
\% \text { reduction in OBE }(\%)\end{array}$ & $23.2(19.6)$ & $22.4(21.0)$ & $\begin{array}{l}6.1(14.8) \\
73.7 \%\end{array}$ & $\begin{array}{l}5.3(9.1) \\
76.3 \%\end{array}$ & $\begin{array}{l}5.6(9.2) \\
75.9 \%\end{array}$ & $\begin{array}{l}8.5(13.7) \\
62.1 \%\end{array}$ \\
\hline $\begin{array}{l}\text { Purging episodes (mean, SD) } \\
\% \text { reduction in purging }(\%)\end{array}$ & $30.6(27.0)$ & $30.5(32.6)$ & $\begin{array}{l}8.3(20.8) \\
72.9 \%\end{array}$ & $\begin{array}{l}7.4(11.5) \\
75.7 \%\end{array}$ & $\begin{array}{l}8.6(15.9) \\
71.9 \%\end{array}$ & $\begin{array}{l}10.1(16.3) \\
66.9 \%\end{array}$ \\
\hline EDE Global (mean, SD) & $3.3(1.1)$ & $3.2(1.1)$ & $1.7(0.9)$ & $1.8(0.9)$ & $1.6(1.1)$ & $1.8(1.0)$ \\
\hline Binge-Purge Abstinence (n, \%) & $1(2.5 \%)$ & $0(0.0 \%)$ & $15(37.5 \%)$ & $9(22.5 \%)$ & $13(32.5 \%)$ & $9(22.5 \%)$ \\
\hline $\begin{array}{l}\text { Global EDE within } 1 \text { SD of community } \\
\text { mean }(n, \%)\end{array}$ & $3(7.5 \%)$ & $6(15.0 \%)$ & $19(47.5 \%)$ & $15(37.5 \%)$ & $22(55.0 \%)$ & $20(50.0 \%)$ \\
\hline \multicolumn{7}{|l|}{ Primary Mechanisms } \\
\hline Ideal Self-discrepancy (mean, SD) & $-0.5(2.7)$ & $-0.3(2.4)$ & $-1.7(2.2)$ & $-1.6(2.4)$ & $-1.8(2.2)$ & $-2.1(1.7)$ \\
\hline Ought Self-discrepancy (mean, SD) & $-0.5(2.2)$ & $-0.8(1.7)$ & $-2.1(1.7)$ & $-1.7(1.5)$ & $-2.3(2.0)$ & $-1.7(1.5)$ \\
\hline DERS Total (mean, SD) & $99.8(27.7)$ & $96.3(25.0)$ & $73.7(24.9)$ & $76.7(21.5)$ & $74.3(28.4)$ & $76.9(22.1)$ \\
\hline \multicolumn{7}{|l|}{ Other outcomes } \\
\hline SBE episodes (mean, SD) & $14.0(18.7)$ & $11.5(13.6)$ & $3.3(4.4)$ & $5.1(7.8)$ & $3.0(6.5)$ & $4.6(7.1)$ \\
\hline EDE Restraint (mean, SD) & $3.0(1.7)$ & $2.7(1.4)$ & $1.3(1.3)$ & $1.2(1.1)$ & $1.2(1.2)$ & $1.2(1.2)$ \\
\hline EDE Eating Concerns (mean, SD) & $2.6(1.2)$ & $2.5(1.4)$ & $0.9(0.7)$ & $1.0(0.7)$ & $0.9(1.0)$ & $1.1(1.1)$ \\
\hline EDE Shape Concerns (mean, SD) & $3.9(1.2)$ & $3.9(1.1)$ & $2.3(1.2)$ & $2.6(1.2)$ & $2.1(1.4)$ & $2.5(1.2)$ \\
\hline EDE Weight Concerns (mean, SD) & $3.8(1.3)$ & $3.7(1.3)$ & $2.3(1.1)$ & $2.4(1.3)$ & $2.1(1.4)$ & $2.2(1.3)$ \\
\hline Undesired Self-discrepancy (mean, SD) & $1.2(2.3)$ & $0.4(1.5)$ & $2.2(2.3)$ & $1.7(2.1)$ & $2.1(2.3)$ & $1.7(2.1)$ \\
\hline BDI Total (mean, SD) & $19.5(11.5)$ & $17.9(11.7)$ & $8.6(8.0)$ & $9.3(9.8)$ & $10.4(11.5)$ & $8.9(9.3)$ \\
\hline RSE Total (mean, SD) & $2.8(1.8)$ & $3.3(1.6)$ & $4.4(1.8)$ & $4.3(1.5)$ & $3.9(2.0)$ & $4.4(1.4)$ \\
\hline SSAI Total (mean, SD) & $46.9(13.3)$ & $45.1(12.5)$ & $35.3(12.5)$ & $35.2(10.1)$ & $35.9(13.7)$ & $37.5(10.8)$ \\
\hline STAI Total (mean, SD) & $52.4(13.6)$ & $50.9(11.3)$ & $38.2(13.2)$ & $39.5(10.0)$ & $39.5(14.2)$ & $41.5(11.4)$ \\
\hline
\end{tabular}

Abbreviations: ICAT =Integrated Cognitive Affective Therapy; CBT-E=Cognitive Behavioral Therapy-Enhanced; $\mathrm{OBE}=$ objective binge eating; $\mathrm{EDE}=$ Eating. 
Table 4. Differences on primary and secondary outcomes and 95\% confidence intervals (CI) between treatments

\begin{tabular}{lrr}
\hline Primary Outcomes & EOT & 4-Month follow-up \\
\hline OBE episodes & $0.30(-2.15$ to 2.75$)$ & $-1.88(-4.53$ to 0.78$)$ \\
Purging episodes & $-0.30(-3.74$ to 3.15$)$ & $0.30(-3.86$ to 4.44$)$ \\
EDE Global & $-0.15(-0.53$ to 0.24$)$ & $-0.25(-0.69$ to 0.19$)$ \\
Primary Mechanisms & & \\
Ideal Self-iscrepancy & & \\
Ought Self-discrepancy & $-0.08(-1.08$ to 0.93$)$ & $0.30(-0.57$ to 1.18$)$ \\
DERS Total & $-0.47(-1.41$ to 0.47$)$ & $-0.55(-1.34$ to 0.23$)$ \\
Secondary Outcomes & $-4.97(-13.15$ to 3.21$)$ & $-4.22(-14.07$ to 5.63$)$ \\
SBE episodes & & \\
EDE Restraint & $-1.22(-3.18$ to 0.75$)$ & $-1.00(-2.73$ to 0.73$)$ \\
EDE Eating Concerns & $0.07(-0.41$ to 0.56$)$ & $-0.15(-0.64$ to 0.35$)$ \\
EDE Shape Concerns & $-0.11(-0.42$ to 0.20$)$ & $-0.27(-0.71$ to 0.18$)$ \\
EDE Weight Concerns & $-0.29(-0.77$ to 0.20$)$ & $-0.37(-0.89$ to 0.16$)$ \\
BDI Total & $-0.16(-0.69$ to 0.37$)$ & $-0.17(-0.74$ to 0.40$)$ \\
RSES Total & $-1.17(-4.75$ to 2.41$)$ & $0.91(-3.19$ to 5.01$)$ \\
SSAI Total & $0.31(-0.37$ to 1.00$)$ & $-0.20(-0.88$ to 0.49$)$ \\
STAI Total & $-0.40(-5.22$ to 4.44$)$ & $-2.37(-7.23$ to 2.50$)$ \\
\end{tabular}

Abbreviations: EOT=End of treatment; ICAT=Integrated Cognitive Affective Therapy; CBT-E=Cognitive Behavioral Therapy-Enhanced; OBE=objective binge eating; $\mathrm{EDE}=$ Eating

${ }^{a}$ Covariate-adjusted estimate (95\% CIs) of difference between ICAT and CBT-E; positive values indicate the estimate for ICAT is higher than the estimate for CBT-E; negative values indicate the estimate for CBT-E is higher than the estimate for ICAT.

${ }^{\mathrm{b}}$ From the Selves Interview.

\section{Reference}

Wonderlich SA, Peterson CB, Crosby RD, Smith TL, Klein MH, Mitchell JE and Crow SJ. A randomized controlled comparison of integrative cognitive-affective therapy (ICAT) and enhanced cognitive-behavioral therapy (CBT-E) for bulimia nervosa. Psychological Medicine, Published online 23 May 2013, doi:10.1017/ S0033291713001098. 